\title{
Spin-entangled currents created by a triple quantum dot
}

\author{
Daniel S. Saraga and Daniel Loss \\ Department of Physics and Astronomy, University of Basel, \\ Klingelbergstrasse 82, CH-4056 Basel, Switzerland
}

\begin{abstract}
We propose a simple setup of three coupled quantum dots in the Coulomb blockade regime as a source for spatially separated currents of spin-entangled electrons. The entanglement originates from the singlet ground state of a quantum dot with an even number of electrons. To preserve the entanglement of the electron pair during its extraction to the drain leads, the electrons are transported through secondary dots. This prevents one-electron transport by energy mismatch, while joint transport is resonantly enhanced by conservation of the total two-electron energy.

PACS numbers : 73.63.-b, 85.35.Be, 3.65.Ud
\end{abstract}

The creation of entangled particles is a crucial problem as entanglement is a prerequisite for quantum computation and communication [1]. While manipulations of entangled photons demonstrating various quantum information processing schemes have been very successful [2], similar achievements are still missing for massive particles such as electrons. Hence there has been a number of theoretical proposals for a solid-state entangler - a device creating two entangled particles and allowing their separation and extraction into two distinct channels for further processing.

Recent proposals involved the extraction of entangled Cooper pairs of a superconductor in contact with quantum dots [3], normal or ferromagnetic conductors 迫, 专], and carbon nanotubes [6, 7]. In another scheme, the entanglement arises from interference effects in a quantum dot in the cotunneling regime and requires special nondegenerate leads of narrow energy width [8]. A generic entangler based on interferometry and which-way detection was proposed in Ref. [9]. In this article, we propose an entangler based on a triple quantum dot setup. The entanglement originates from the singlet state of a pair of electrons in one quantum dot, while its transport relies on energy filtering by secondary dots. Our proposal is based on existing technology [10] and on realistic parameter values as typically found in transport experiments with quantum dots.

Setup. Fig. 1 describes the proposed entangler. It is composed of three coupled lateral quantum dots $\left(D_{C}, D_{L}\right.$ and $\left.D_{R}\right)$ in the Coulomb blockade regime, each of them coupled to a Fermi liquid lead $l_{C}, l_{L}$ and $l_{R}$. When two excess electrons are present in $D_{C}$, we can assume [11] that their ground state is the spin-singlet state, which is the (anti)symmetric superposition of their (spin) wavefunctions. The aim of the entangler is to extract the singlet from $D_{C}$, by transporting one electron into the neighboring $\operatorname{dot} D_{L}$ and the other one into $D_{R}$, and finally transport them into the drain leads $l_{L}$ and $l_{R}$ without loss of entanglement. This creates two currents of pairwise spin-entangled electrons that are spatially separated.

Applying two bias voltages $\mu_{C}-\mu_{L}$ and $\mu_{C}-\mu_{R}$ allows the transport of electrons from the source lead $l_{C}$

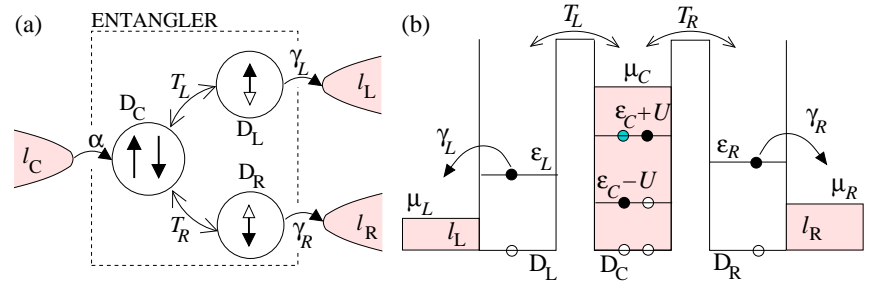

Figure 1: (a) Setup of the triple quantum dot entangler. Three leads $l_{i}(i=C, L, R)$ at chemical potential $\mu_{i}$ are coupled to three quantum dots $D_{i}$ in the Coulomb blockade regime. Each dot contains an even number of electrons, and can only accept $0,1\left(D_{L}\right.$ and $\left.D_{R}\right)$ and $2\left(D_{C}\right)$ excess electrons. A spin-singlet is formed in $D_{C}$ when two electrons tunnel incoherently, each with a rate $\alpha$, from the source lead $l_{C}$ into $D_{C}$. Each of the electrons can subsequently tunnel coherently to $D_{L}$ and $D_{R}$ with tunneling amplitudes $T_{L}$ and $T_{R}$. Finally, the electrons tunnel out (with rates $\gamma_{L}$ and $\gamma_{R}$ ) to the drain leads $l_{L}$ and $l_{R}$, creating two currents of entangled electrons. (b) Energy level diagram, for each electron. Non-entangled currents, which arise from one-electron transport, are suppressed by the energy differences $\epsilon_{L, R}-\epsilon_{C} \pm U$. The joint transport of both electrons is favored by conservation of the total two-electron energy: $\epsilon_{L}+\epsilon_{R} \simeq 2 \epsilon_{C}$ (resonance condition).

to both drain leads $l_{L}$ and $l_{R}$, via the three quantum $\operatorname{dots} D_{i}, i=C, L, R$. To preserve the entanglement of the electrons until they are in the drain leads, one must avoid the individual transport of one electron, as this would allow the arrival of a new electron in $D_{C}$ which could destroy the existing entanglement by forming a new singlet with the remaining electron. To suppress one-electron transport, we arrange the dots so that there is a large difference between the energy levels of $D_{L}$ and $D_{C}$ compensated by the energy difference between $D_{R}$ and $D_{C}$. This way the joint transport of both electrons to each neighboring dot conserves energy and is therefore enhanced by resonance, while the off-resonant transport of one electron is suppressed by the energy difference.

The number of electrons participating in the transport is controlled via Coulomb blockade [12], where $N$ excess electrons in dot $D_{i}$ create a large electrostatic Coulomb charging energy $U_{i}(N)$. The energy of the $N^{\text {th }}$ electron is then $E_{i}(N)=U_{i}(N)-U_{i}(N-1)+\epsilon_{i}(N)$, where $\epsilon_{i}(N)$ is the lowest single-particle energy available for the $N^{\text {th }}$ electron. We consider the electrons to be independent and neglect further effects such as interdot charging en- 
ergy or exchange Coulomb interaction. Assuming a shell filling of each dot [12], we disregard all but the excess electrons in each dot. The ground state in $D_{C}$ with 2 excess electrons is the spin singlet $\uparrow \downarrow-\downarrow \uparrow$, where both electrons have the same orbital energy $\epsilon_{C}=\epsilon_{C}(1)=\epsilon_{C}(2)$. One excess electron in $D_{L}$ or $D_{R}$ cannot form a singlet with one of the electrons already present as these are already all paired up in singlets. We assume the gate voltages of each dot so that $U_{j}(0)=U_{j}(1), j=L, R$ and $U_{C}(0)=U_{C}(2)=0$, which gives a negative charging energy for one electron: $U_{C}(1)=-U$. We define the zero energy as the total energy of the three empty dots.

The energy levels in the dots and the chemical potentials $\mu_{i}$ in the leads are assumed to be tuned such that only zero or one excess electron in $D_{j}, j=L, R$, and zero, one, or two electrons in $D_{C}$ are allowed. It is crucial that only the ground states of the electronic levels in the dots participate in the transport. In particular, the triplet states in $D_{C}$ should not be accessible to incoming electrons. To avoid resonance with excited levels, the energy level spacings in the dots must be larger than the Coulomb charging energies: $\Delta \epsilon_{i}>U_{i}$. Excited states with energy $E_{i}^{*}$ could participate in the transport through cotunneling events [12], where one describes the transport of one electron from $l_{C}$ to $l_{j}$ via intermediate "virtual states" in the dots by second-order processes. We shall neglect such events, as they are suppressed by factors of the order of $\alpha /\left(E_{i}^{*}-\mu_{i}\right) \simeq \alpha /\left(U_{i}-\mu_{i}\right) \ll 1$.

We need low temperatures $T$ so that thermal fluctuations cannot allow three electrons in $D_{C}$ or populate excited levels, which could also create a current in the reverse direction $\left(l_{j} \rightarrow D_{j}, D_{C} \rightarrow l_{C}\right)$. Taking $k_{\mathrm{B}} T \ll\left|\mu_{i}-E_{i}(0,1,2,3)\right|$, we can neglect temperature effects and set $T=0$ for simplicity. The incoherent tunneling rates are given by $\alpha=2 \pi\left|t_{C}\right|^{2} \nu_{C}$ and $\gamma_{j}=2 \pi\left|t_{j}\right|^{2} \nu_{j}$, where $t_{i}$ is the tunneling matrix element connecting $l_{i}$ and $D_{i}$, and $\nu_{i}$ is the density of states of the lead.

The quantum states of the entangler are given by combining the different numbers of electrons allowed in each dot. 0 describes the situation where all the dots are empty; $L, R$ or $C$ corresponds to one electron in $D_{L}, D_{R}$ or $D_{C}$, while $C C$ denotes the singlet state created by two electrons in $D_{C}$. Thus, the 8 states, shown in Fig. 2 with their transitions, for the basis set $\mathcal{B}=\{0, C, C C, L C, C R, L R, L, R\}$. This description in terms of the individual levels of each isolated dot requires that the tunneling matrix elements $T_{L}$ and $T_{R}$ (considered to be real) connecting the dots are small and do not mix the levels in different dots, i.e. $T_{L}, T_{R} \ll \Delta \epsilon_{i}$.

The coherent evolution is described by a Hamiltonian matrix $H_{k, k^{\prime}}, k, k^{\prime} \in \mathcal{B}$. The diagonal elements contain the energies $E_{0}=0, E_{C}=\epsilon_{C}-U, E_{C C}=2 \epsilon_{C}, E_{L C}=$ $\epsilon_{L}+\epsilon_{C}-U, E_{C R}=\epsilon_{R}+\epsilon_{C}-U, E_{L R}=\epsilon_{L}+\epsilon_{R}, E_{L}=$ $\epsilon_{L}, E_{R}=\epsilon_{R}$, while the off-diagonal elements describe the coherent oscillations of one electron between $D_{C}$ and $D_{L}$ or $D_{R}: H_{C, L}=H_{C R, L R}=T_{L}, H_{C, R}=H_{L C, L R}=$

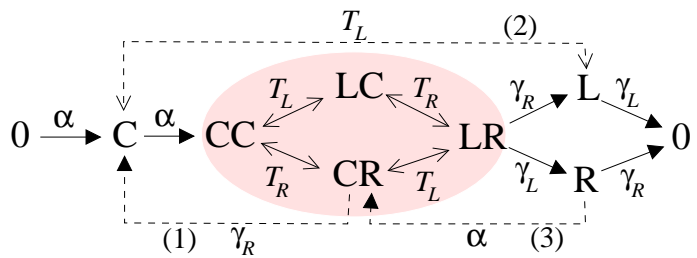

Figure 2: The entangler states $\{0, C, C C, L C, C R, L R, L, R\}$ and their transitions. Double arrows indicate coherent tunneling (oscillations) of one electron between two dots with overlap matrix element $T_{L}$ and $T_{R}$ (in the shaded area). Single arrows indicate incoherent tunneling from $(\alpha)$ or to $\left(\gamma_{L}\right.$ and $\left.\gamma_{R}\right)$ the leads. The dashed lines indicate the three types of transitions that must be avoided to ensure the joint transport of the singlet pair $C C$ to the leads (see text; for clarity we do not show the transitions obtained by replacing $L$ by $R$.)

$T_{R}, H_{C C, L C}=T_{L} \sqrt{2}$ and $H_{C C, C R}=T_{R} \sqrt{2}$. The $\sqrt{2}$ factor comes from the identical orbital states in $C C$.

We describe the incoherent transport as sequential tunneling (lowest order in $\alpha, \gamma_{L}, \gamma_{R}$ ) in terms of the master equation for the (reduced) density matrix $\rho$ of the entangler. The diagonal elements, $\rho_{k}, k \in$ $\mathcal{B}$, are the occupation probabilities of the state $k$, with normalization $\sum_{k} \rho_{k}=1$. The off-diagonal elements $\rho_{k, k^{\prime}}$ contain the coherent superposition of $k$ and $k^{\prime}$. We write the master equation 13 as $\mathrm{d} \rho / \mathrm{dt}=$ $-i[H, \rho]-M$, where $M$ describes the incoherent transport from/to a lead connecting a state $k$ to another state $k^{\prime}$ with a rate $W\left(k^{\prime}, k\right) \in\left\{\alpha, \gamma_{L}, \gamma_{R}\right\}$. For the diagonal elements, this results in the population equation: $M_{k}=-\rho_{k} \sum_{k^{\prime}} W\left(k^{\prime}, k\right)+\sum_{k^{\prime}} W\left(k, k^{\prime}\right) \rho_{k^{\prime}}$, while the off-diagonal elements are damped by the incoherent transitions out of each of the two corresponding states: $M_{k, k^{\prime}}=-\frac{1}{2} \rho_{k, k^{\prime}} \sum_{k^{\prime \prime} \neq k, k^{\prime}} W\left(k^{\prime \prime}, k\right)+W\left(k^{\prime \prime}, k^{\prime}\right)$. Out of the 64 elements of $\rho$, only 24 are coupled to the relevant diagonal elements. Arranging them in a real vector $\vec{V}$, one can rewrite the master equation as a homogeneous first-order differential equation given by a matrix $\mathcal{A}: \mathrm{d} \vec{V} / \mathrm{dt}=\mathcal{A} \vec{V}$. Its stationary solution is the eigenvector corresponding to the zero eigenvalue of $\mathcal{A}$. It can be found symbolically by a mathematical software (MAPLE), and defines the stationary populations $P_{k}=\rho_{k}^{0}$ of the different states $k$ of the entangler.

Results. Our aim is to show that the transport of the singlet state through the entangler is robust and dominates over the transport of uncorrelated electrons. We define the stationary entangled current (see the central sequence in Fig. 2) as $I_{E}=e \gamma_{L}\left(P_{L R}+P_{L}\right)+e \gamma_{R}\left(P_{L R}+\right.$ $\left.P_{R}\right)$. The destruction of the entanglement of the two electrons can occur in three ways; see Fig. 2 (the following discussion also refers to the cases with $L$ replaced by $R$ ).

(1) An electron tunnels out from $D_{R}$ to $l_{R}$ while the second electron is still in $D_{C}$. This creates a current $e \gamma_{R} P_{C R}$ which might contain no entanglement, as the remaining electron can form a new singlet with a new electron coming from $l_{C}$. This current is suppressed by 
the energy mismatch $\epsilon_{R}-\epsilon_{C}-U$ between the states $C C$ and $C R$. The entangled current $I_{E}$ is dominant if the entangler quality $Q=\min \left\{P_{L R} / P_{C R}, P_{L R} / P_{L C}\right\}$ satisfies $Q \gg 1$.

(2) An electron tunnels from $D_{C}$ to $D_{L}$ and then out to $l_{L}$ before a second electron has tunneled into $D_{C}$ and formed a singlet. This channel is suppressed by the energy difference $\epsilon_{L}-\epsilon_{C}+U$ between the states $C$ and $L$. As the stationary solution contains no information on the history of the electrons, we compare currents obtained in the two following cases. (i) We switch off the undesired channel by setting $T_{L}=T_{R}=0$ between $C, L$ and $R$, while keeping the tunneling between $C C, C R, L C$, and $L R$. This defines the stationary populations $P_{k}$. (ii) We keep the undesired channel, while switching off the tunneling involving $C C$ and $L R$. This defines the populations $\tilde{P}_{k}$, and creates a current $e \gamma_{L}\left(\tilde{P}_{L}+\tilde{P}_{L C}\right)+e \gamma_{R}\left(\tilde{P}_{R}+\tilde{P}_{C R}\right)$ containing no entanglement. Defining a second entangler quality by $\widetilde{Q}=\min \left\{P_{L} / \tilde{P}_{L}, P_{L R} / \tilde{P}_{L C}, P_{R} / \tilde{P}_{R}, P_{L R} / \tilde{P}_{C R}\right\}$, the condition $\widetilde{Q} \gg 1$ corresponds to the suppression of this one-electron channel.

(3) After the joint transport of the two electrons into state $L R$ and the tunneling of one electron into $l_{L}$, a new electron tunnels into $D_{C}$ before the remaining electron in $D_{R}$ has tunneled out to $l_{R}$. The new electron can then form a new singlet with the remaining electron, therefore destroying the entanglement that existed with the electron which has moved to the lead $l_{L}$. To suppress this channel, we need $\alpha \ll \gamma_{L}, \gamma_{R}$. For simplicity, we set $\alpha=0$ for transitions from $L$ and $R$ when calculating the probabilities $P_{k}$ (but we keep $\alpha$ non-zero for $\tilde{P}_{k}$ ).

The exact analytical expressions for $P_{k}$ are extremely lenghty and cannot be written in a compact form. We introduce $\delta \epsilon_{j}=\epsilon_{j}-\epsilon_{C}$, which explicitly removes the dependence on $\epsilon_{C}$ as only energy differences enter the time evolution of the density matrix $\rho(t)$. Secondly, we consider a symmetric setup with $\gamma=\gamma_{L}=\gamma_{R}$ and $T_{0}=T_{L}=T_{R}$. Then, the symmetry between $L R, L$, and $R$ yields $P_{L}=P_{R}=P_{L R}$ (see Fig. 2). Thirdly, we consider the case $\epsilon_{R}=\epsilon_{C}$. The resonance condition for the joint transport $E_{C C}=2 \epsilon_{C} \simeq E_{L R}=\epsilon_{L}+\epsilon_{R}$ translates into $\delta \epsilon_{L} \simeq 0$, and the energy differences relevant for the suppression of one-electron transport are given by $U$ and $U \pm \delta \epsilon_{L}$. From a qualitative analysis involving first and second-order perturbation calculations (à la Fermi Golden Rule), we find that the conditions for a dominant joint transport read $\alpha \ll \gamma \ll T_{0} \ll U,\left|U \pm \delta \epsilon_{L}\right|$ 114. Hence we expand both the numerator and denominator of the expressions for $P_{k}$ in the lowest non-trivial order in $\alpha, \gamma, T_{0}$. We distinguish two cases:

[I] non-resonant $\left(\left|\delta \epsilon_{L}\right|>T_{0}\right)$ :

$$
P_{L}=P_{R}=P_{L R} \simeq \frac{2 T_{0}^{4}\left(2 U-\delta \epsilon_{L}\right)^{2}}{\delta \epsilon_{L}^{2} U^{2}\left(U-\delta \epsilon_{L}\right)^{2}}
$$

$$
\begin{aligned}
P_{C R} & \simeq \frac{2 T_{0}^{2}}{U^{2}}, P_{L C} \simeq \frac{2 T_{0}^{2}}{\left(U-\delta \epsilon_{L}\right)^{2}} \\
\tilde{P}_{R} & \simeq \frac{T_{0}^{2}}{U^{2}}, \quad \tilde{P}_{L} \simeq \frac{T_{0}^{2}}{\left(U+\delta \epsilon_{L}\right)^{2}}
\end{aligned}
$$

[II] resonant $\left(\delta \epsilon_{L}=0\right)$ :

$$
\begin{aligned}
& P_{L}=P_{R}=P_{L R} \simeq \frac{8 \alpha T_{0}^{4}}{\alpha \gamma^{2} U^{2}+4 T_{0}^{4}(8 \gamma+9 \alpha)}, \\
& P_{C R}=P_{L C} \simeq \frac{2 \alpha T_{0}^{2}\left(\gamma^{2} U^{2}+40 T_{0}^{4}\right)}{U^{2}\left[\alpha \gamma^{2} U^{2}+4 T_{0}^{4}(8 \gamma+9 \alpha)\right]}, \\
& \tilde{P}_{R}=\tilde{P}_{L} \simeq \frac{\alpha \gamma T_{0}^{2}}{\alpha \gamma U^{2}+2 T_{0}^{2}(\gamma+\alpha)^{2}},
\end{aligned}
$$

and $\tilde{P}_{C R}=\tilde{P}_{R} \alpha / \gamma, \tilde{P}_{L C}=\tilde{P}_{L} \alpha / \gamma$.

These two cases are sufficient for an analytical discussion of the entangler, as the approximate expressions (11)(6) reproduce accurately the exact results presented in Fig. 3. As shown in Fig. 3(a), the entangler qualities $Q$ and $\widetilde{Q}$ reach a maximum around $\delta \epsilon_{L}=0$, which is due to a resonance in the coherent oscillations between $C C$ and $L R$. We define now the quantities $Q_{\mathrm{I}}$ and $\widetilde{Q}_{\mathrm{I}}$ as approximations of the qualities $Q$ and $\widetilde{Q}$ obtained with Eqs. (1])-(3). $Q_{\mathrm{I}}$ and $\widetilde{Q}_{\mathrm{I}}$, which grow as $\left(\delta \epsilon_{L}\right)^{-2}$, give a correct estimate of the width $\overline{\delta \epsilon_{L}}$ of the
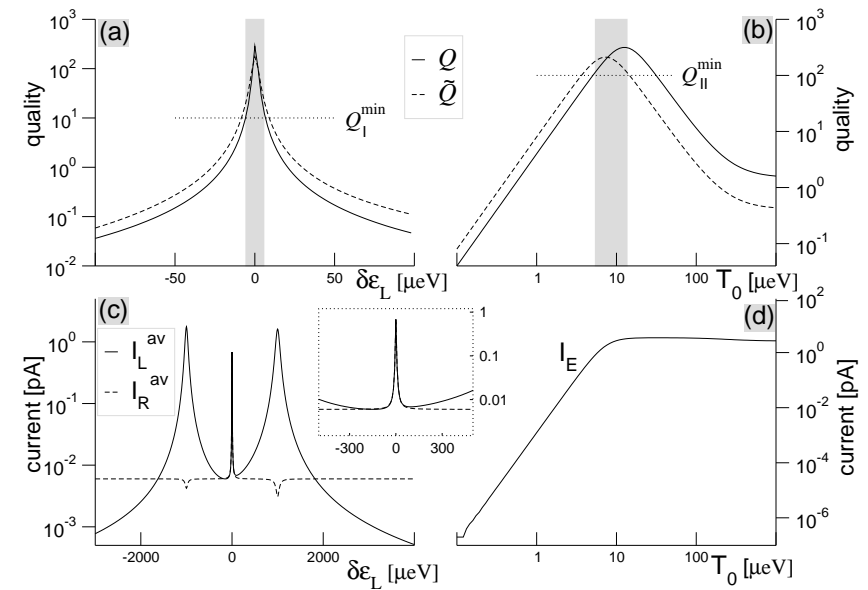

Figure 3: Quality of the entangler and output current, with the parameters $\alpha=0.1, \gamma=1, T_{0}=10, U=1000$ in $\mu \mathrm{eV}$. (a) Quality $Q$ and $\widetilde{Q}$, around the resonance at $\delta \epsilon_{L}=0$ where the entangled current dominates. The width of the resonance defined by $Q, \widetilde{Q}>Q_{\mathrm{I}}^{\min }=10$ is $\left|\delta \epsilon_{L}\right|<\overline{\delta \epsilon_{L}}=2 T_{0} / \sqrt{Q_{\mathrm{I}}^{\min }} \simeq$ $6 \mu \mathrm{eV}$ (shown in gray). (b) $Q$ and $\widetilde{Q}$ as a function of the tunneling matrix element $T_{0}$ at resonance $\left(\delta \epsilon_{L}=0\right)$. In gray, the region where the quality of the entangler is $Q, \widetilde{Q}>Q_{\mathrm{II}}^{\min }=$ 100. (c) Average current in the left $\left(I_{L}^{\mathrm{av}}\right)$ and in the right $\left(I_{R}^{\text {av }}\right)$ leads, which takes into account both entangled and nonentangled currents. A symmetric current $\left(I_{L}^{\mathrm{av}}=I_{R}^{\mathrm{av}}\right)$ is the signature of the resonance and therefore of the desired regime where the entangled currents dominate (see the inset with a larger scale). (d) At resonance, the entangled current $I_{E}$ saturates to $\simeq \alpha=4 \mathrm{pA}$ when $T_{0} \gg\left(U^{2} \gamma \alpha / 32\right)^{1 / 4} \simeq 5 \mu \mathrm{eV}$. 
resonance around $\delta \epsilon_{L}=0$. Introducing the condition $Q_{\mathrm{I}}, \widetilde{Q}_{\mathrm{I}}>Q_{\mathrm{I}}^{\min }$, we get $\left|\delta \epsilon_{L}\right|<\overline{\delta \epsilon_{L}}=2 T_{0} / \sqrt{Q_{\mathrm{I}}^{\min }}$. Note that such proportionality of the width to the tunneling matrix element is also found in the Rabi formula for a two-level system. Similarly, we define $Q_{\text {II }}$ and $\widetilde{Q}_{\mathrm{II}}$ with Eqs. (4)-(6); these approximate ratios accurately reproduce the height of the resonance peak at $\delta \epsilon_{L}=0$. Introducing the condition $Q_{\mathrm{II}}, \widetilde{Q}_{\mathrm{II}}>Q_{\mathrm{II}}^{\min }$, we find $\gamma \sqrt{Q_{\mathrm{II}}^{\min } / 8}<T_{0}<U \sqrt{4 \alpha / \gamma Q_{\mathrm{II}}^{\min }}$; see Fig. 3(b).

Quantities which are experimentally accessible are the currents in the left and right leads, $I_{L}=e \gamma_{L}\left(P_{L C}+\right.$ $\left.P_{L R}+P_{L}\right)$, and $I_{R}=e \gamma_{R}\left(P_{C R}+P_{L R}+P_{R}\right)$. Far from resonance $\left(\delta \epsilon_{L}>T_{0}\right)$, one cannot neglect the currents $\tilde{I}_{L}$ and $\tilde{I}_{R}$ coming from the single-electron transitions described by the probabilities $\tilde{P}_{k}$. Hence we consider in Fig. 3(c) the average of the currents in situations (i) and (ii): $I_{L}^{\mathrm{av}}=\left(I_{L}+\tilde{I}_{L}\right) / 2$ and $I_{R}^{\mathrm{av}}=\left(I_{R}+\tilde{I}_{R}\right) / 2$. Away from the two-electron resonance the current is asymmetric: $I_{L}^{\mathrm{av}} \neq I_{R}^{\mathrm{av}}$. The large peaks where $I_{L}^{\mathrm{av}} \gg I_{R}^{\mathrm{av}}$ are due to one-electron resonances: between $C C$ and $L C$ when $\delta \epsilon_{L}=U$ (right peak), and between $C$ and $L$ when $\delta \epsilon_{L}=-U$ (left peak). At the $\delta \epsilon_{L}=0$ resonance the current is symmetric as the electrons are transported together and simultaneously from the central dot to the leads [see inset in Fig. 3(c)]. Hence by varying $\epsilon_{L}$ via the gate voltage of $D_{L}$ until $I_{L}=I_{R}$, one can locate the resonance, and therefore the regime where the entangler is most efficient [15]. Finally, Eq. (4) gives $P_{L R} \rightarrow \alpha / 4 \gamma$ for $T_{0}^{4} \gg U^{2} \gamma \alpha / 32$, which yields $I_{E} \rightarrow I_{\max }=\alpha$, as illustrated in Fig. 3(d). Note that in order to be able to reach $I_{\max }$ within the window $\gamma \sqrt{Q_{\mathrm{II}}^{\min } / 8}<T_{0}<U \sqrt{\alpha / 4 \gamma Q_{\mathrm{II}}^{\min }}$, one also needs the condition $U>Q_{\mathrm{II}}^{\min } \gamma \sqrt{\gamma / 2 \alpha}$.

Discussion. Setting the qualities of the entangler to $Q_{\mathrm{II}}^{\min }=100, Q_{\mathrm{I}}^{\min }=10$ and $\gamma=10 \alpha$, we need, approximately, $35 \alpha<T_{0}<U / 60$, and $U>2200 \alpha$. The first condition is easily met as $\alpha$ and $T_{0}$ can be varied via the voltages defining the barriers [16]. For the second condition, present-day experiments manipulate currents typically around $2 \mathrm{pA}\|16\|$, yielding $\alpha>0.1 \mu \mathrm{eV} \Rightarrow U>0.3 \mathrm{meV}$. To get a finite width $\delta \epsilon_{L} \simeq 6 \mu \mathrm{eV}$, we take $U \simeq 1 \mathrm{meV}$, which is within reported values $[12$, 16]. To obtain an even better quality $\left(Q_{\mathrm{II}}^{\min }=1000, \gamma=100 \alpha\right)$, one needs to increase the ratio $U / \alpha$. One possible issue is the singleparticle energy spacing $\Delta \epsilon_{i}$, which is usually smaller than or equal to the charging energy. However, as $\Delta \epsilon_{i} \propto 1 / L^{2}$ in a box of size $L$, while $U \propto 1 / L$, one should be able to reach $\Delta \epsilon_{i} \gg U$ by decreasing the dot size. Alternatively, one can use vertical quantum dots, which have large energy level spacings [12], or use one carbon nanotube with two bendings (which defines three regions behaving like quantum dots).

A current $I_{\max } \simeq 10 \mathrm{pA}$ corresponds to the delivery of an entangled pair every $t_{\mathrm{p}} \simeq 1 / \alpha \simeq 16 \mathrm{~ns}$, which is below reported spin decoherence time of $100 \mathrm{~ns}$ |17|. The av- erage separation between two entangled electrons is approximately $t_{\mathrm{e}} \simeq 1 / U \simeq 0.4 \mathrm{ps} \ll t_{\mathrm{p}}$, with a maximal separation of $t_{\mathrm{m}} \simeq 1 / \gamma \simeq 0.5 \mathrm{~ns}$. This would allow noise measurements using a beam splitter, where an enhancement in the two-terminal noise is a signature of singlet states compared to the noise of non-correlated electrons 181. Note that such an experiment requires electrons with the same orbital energy, which can be achieved if $\epsilon_{L}=\epsilon_{R}=\epsilon_{C}$. One could also carry out a measurement of Bell's inequalities by measuring the spin of the electron in each lead with the help of a spin filter based on a quantum dot [19.

We thank H.A. Engel, P. Recher, C. Egues, and G. Burkard for useful discussions. Financial support from NCCR "Nanoscale Science", the Swiss NSF, DARPA, and ARO is gratefully acknowledged.

[1] C.H. Bennett and D.P. DiVincenzo, Nature (London) 404, 247 (2000).

[2] W. Tittel and G. Weihs, Quantum Information and Computation 1, 3 (2001), and references therein.

[3] P. Recher, E.V. Sukhorukov, and D. Loss, Phys. Rev. B 63, 165314 (2001).

[4] G.B. Lesovik, T. Martin, and G. Blatter, Eur. Phys. J. B 24, 287 (2001).

[5] R. Mélin, cond-mat/0102073; G. Falci, D. Feinberg, and F.W.J. Hekking, Europhys. Lett. 54, 255 (2001).

[6] P. Recher and D. Loss, Phys. Rev. B 65, 165327 (2002).

[7] C. Bena, S. Vishveshwara, L. Balents, and M.P.A. Fisher, cond-mat/0202102.

[8] W.D. Oliver, F. Yamaguchi, and Y. Yamamoto, Phys. Rev. Lett. 88, 037901 (2002).

[9] S. Bose and D. Home, Phys. Rev. Lett. 88, 050401 (2002).

[10] F.R. Waugh et al., Phys. Rev. Lett. 75, 705 (1995).

[11] S. Tarucha et al., Phys. Rev. Lett. 84, 2485 (2000). It might be necessary to apply a small magnetic field or to distort the confining potential to lift the orbital degeneracy which could favor the triplet state corresponding to Hund's rule. An alternative is to start with an empty dot, where the two-electron ground-state is a singlet.

[12] L.P. Kouwenhoven et al., in Mesoscopic Electron Transport, NATO ASI Series E, Vol. 345 (Kluwer, Dordrecht, 1997).

[13] K. Blum, Density Matrix Theory and Applications (Plenum, New York, 1996).

[14] Although the requirement $\gamma \ll T_{0}$ is not consistent with the conditions of validity of a perturbation theory in $T_{0}$, such a calculation clarifies the physical picture of the entangler by showing explicitly the suppression due to the energy mismatch.

[15] The entangled current has a small asymmetry $(\sim 5 \%)$ at resonance if $\gamma_{L} \neq \gamma_{R}, T_{L} \neq T_{R}$.

[16] T.H. Oosterkamp et al., Nature (London) 395, 873 (1998); T. Fujisawa et al., Science 282, 932 (1998).

[17] J.M. Kikkawa and D.D. Awschalom, Phys. Rev. Lett. 80, 4313 (1998).

[18] G. Burkard, D. Loss, and E.V. Sukhorukov, Phys. Rev. B 61, R16303 (2000).

[19] P. Recher, E.V. Sukhorukov, and D. Loss, Phys. Rev. Lett. 85, 1962 (2000). 